\title{
Management of pediatric tonsillectomy pain: a review of the literature
}

This article was published in the following Dove Press journal:

Ambulatory Anesthesia

12 May 2016

Number of times this article has been viewed

\author{
Jennifer Hansen \\ Ravi D Shah \\ Hubert A Benzon \\ Department of Pediatric \\ Anesthesiology, Ann and Robert $\mathrm{H}$. \\ Lurie Children's Hospital of Chicago, \\ Northwestern University Feinberg \\ School of Medicine, Chicago, IL, USA
}

Correspondence: Hubert A Benzon Department of Pediatric Anesthesiology, Ann and Robert H. Lurie Children's Hospital of Chicago, 225 E Chicago Avenue, Box 19, Chicago, IL 606II, USA

Tel + I 3122275170

$\mathrm{Fax}+$ I 3122279730

Email hbenzon@luriechildrens.org
Abstract: Tonsillectomy is associated with significant pain and postoperative pain control is often unsatisfactory. We discuss the various strategies that have been investigated to control pain following tonsillectomy. Codeine is a weak analgesic frequently used in children for the treatment of mild-to-moderate pain, however, due to adverse events related to its metabolism, it has been contraindicated for postoperative pain in children since 2013. Intravenous morphine is frequently used for moderate-to-severe pain in children, however, its active metabolite can lead to respiratory depressant and other undesirable side effects. Hydromorphone is a commonly used alternative that has been studied infrequently. Alternatives to narcotic pain strategies have also been studied. Nonsteroidal anti-inflammatory drugs (NSAIDs) are effective as analgesics, yet many practitioners avoid their use given the concern for postoperative bleeding. Intraoperative acetaminophen has been shown to improve postoperative pain and decrease recovery room time. Dexamethasone has been shown to improve postoperative pain, vomiting, and decrease airway swelling, and seems to be effective for use during tonsillectomy surgery. Ketamine has been shown to decrease analgesic requirements without adverse affects of hallucinations. Direct injection of local anesthetic into the tonsillar bed has been shown to be effective in improving pain control, however, there is concern that local anesthetic could be erroneously injected into the carotid artery and lead to devastating consequences. Optimal pain control regimens following pediatric tonsillectomy continue to be a challenge for both anesthesiologists and otorhinolaryngologists. Opioids are the most commonly used but are associated with significant side effects, especially postoperative respiratory depression. Adjuncts may be safely used in the perioperative period and may have narcotic sparing effects as well as improved cost effectiveness. Further research into additional analgesics strategies is necessary to improve the perioperative course.

Keywords: pediatric, pain, tonsillectomy

\section{Introduction}

Tonsillectomy is one of the most commonly performed operations in children in the US, with over half a million tonsillectomies performed in the US in children aged under 15 per year. $^{1}$ Tonsillectomies in children are associated with significant morbidity and rarely, mortality. Common complications following tonsillectomy include respiratory depression, inadequate pain control, hypovolemia secondary to decreased oral intake, and postoperative hemorrhage. ${ }^{2}$

Tonsillectomy (with or without adenoidectomy) is associated with significant pain and postoperative pain control is often unsatisfactory, frequently persisting beyond the seventh postoperative day. Inadequate pain control may lead to decreased oral intake, readmission for pain control, and continued pathologic responses to pain may 
lead to chronic pain in adulthood. ${ }^{3}$ Opioid analgesics, while previously considered first-line therapy, may be associated with inadequate analgesia and side effects such as nausea, vomiting, pruritus, decreased gastrointestinal motility, sedation, and respiratory depression.

Up to $80 \%$ of tonsillectomies are performed for sleepdisordered breathing. ${ }^{4}$ The population undergoing tonsillectomy is often young and presents with obstructive symptoms, potentially leading to hypoxic episodes postoperatively. This population has been shown to be particularly sensitive to the respiratory depressant effects of morphine. ${ }^{5}$ Recent black-box warnings on codeine use due to variable pharmacodynamics have resulted in changes in standard of care and investigations into nonnarcotic and multimodal analgesic regimens. ${ }^{6}$ Here we discuss the various strategies that have been investigated to more adequately and safely control pain following tonsillectomy.

\section{Discussion}

Codeine is a weak analgesic frequently used in children for the treatment of mild-to-moderate pain. ${ }^{7}$ Morphine, the product of codeine metabolism via cytochrome P450 2D6 (CYP2D6) is the primary agent responsible for the analgesic effects of codeine. ${ }^{7}$ The quantity of morphine produced from this reaction is highly variable and phenotype-dependent. Four phenotypes have been described: poor metabolizer (PM), intermediate metabolizer (IM), extensive metabolizer (EM), and ultrarapid metabolizer (UM). ${ }^{7}$ While PM's produce very limited morphine and often experience inadequate pain relief, UM's produce increased amounts of morphine and are at risk of opioid intoxication. US Food and Drug Administration (FDA) reviewed data of adverse events in children from 1969 to May 2012 and found 13 cases of codeine-related adverse events, ten of which resulted in death. ${ }^{8}$ The majority of these cases were following tonsillectomy and children ranged from 21 months to 9 years of age. Of the seven children undergoing genetic analysis, three were found to be ultrafast metabolizers and one probably ultrafast metabolizer. ${ }^{8}$ In February 2013, the FDA declared that codeine is contraindicated for postoperative analgesia use in children.

Morphine is a frequently used analgesic for moderateto-severe pain in children. While widely used intravenously during the perioperative period, oral morphine for postoperative pain has poor bioavailability, a narrow therapeutic index and large interindividual variability in plasma levels following intake. ${ }^{6}$ Morphine is metabolized into morphine-3-glucuronide and morphine-6-glucuronide. Morphine-6-glucuronide is an active metabolite that is more potent than morphine with both analgesic and respiratory depressant effects and may accumulate with repeated dosing. Oral morphine increased the risk of postoperative oxygen desaturation compared with ibuprofen in a recent study with no difference in analgesic effectiveness or posttonsillar hemorrhage rates. ${ }^{9}$ Given the large individual variability, rates of oxygen desaturation and lack of improvement in pain control compared to ibuprofen, morphine is likely not an effective replacement for codeine.

Hydromorphone may offer an analgesic advantage over morphine, however, not an improved side effect profile with respect to nausea, vomiting, or itching. ${ }^{10}$ However, studies specific to hydromorphone in comparison to morphine specifically for pediatric tonsillectomy are lacking. Hydromorphone is also associated with a high number of medication errors due to errors in conversion of equipotent doses from morphine. ${ }^{6}$

Nonsteroidal anti-inflammatory drugs (NSAIDs) are effective analgesics, however, their use is controversial following tonsillectomy given the concern for bleeding. Ketorolac (0.5 mg/kg max up to $30 \mathrm{mg}$ intravenous [IV]) use in particular has been associated with posttonsillar hemorrhage, with rates ranging from $4.4 \%$ to $18 \% .{ }^{11} \mathrm{~A}$ recent retrospective chart review revealed increased rates of posttonsillar hemorrhage requiring return to operating room in children who received ibuprofen plus acetaminophen (1.6\%) versus narcotic plus acetaminophen $(0.5 \%) .{ }^{12}$ However, this is conflicting with a Cochrane review, with over 900 children from randomized control trials, which found that NSAIDs did not significantly alter postoperative bleeding compared with placebo or other pain management strategies. ${ }^{11}$ IV ibuprofen given on induction has been shown to decrease dosage of rescue fentanyl in the recovery period with no significant difference in surgical blood loss or incidence of postoperative rebleeding. ${ }^{13}$ As previously mentioned, oral ibuprofen (4-10 mg/kg) when combined with acetaminophen was associated with effective analgesia and less desaturation events on the first postoperative night than morphine combined with acetaminophen. ${ }^{9}$ These findings demonstrate that NSAIDs, excluding ketorolac, can be used safely and effectively in the perioperative period and may have a narcotic-sparing effect. However, the decision to give an NSAID intraoperatively requires discussion with the surgical team involved, and surgeons may be hesitant to administer medications that can lead 
to potential postoperative complications such as bleeding. In our practice, we typically do not administer ketorolac perioperatively for posttonsillectomy pain.

IV acetaminophen is indicated to treat mild-to-moderate postoperative pain in children. It has been shown to improve postoperative pain control as well as shorten post-anesthesia care unit (PACU) stay by $\sim 10$ minutes. ${ }^{14} \mathrm{~A}$ recent study demonstrated that the combination of IV acetaminophen and opioids are more effective in preventing need for rescue analgesia and more cost-effective. ${ }^{4}$ Although the medication cost of IV acetaminophen is higher, the shortened postanesthesia recovery unit stay and decreased need for rescue analgesia was proven to be cost-effective. While useful in conjunction with opioids, acetaminophen alone may not provide adequate analgesia. ${ }^{11}$ Postoperatively, scheduled dosing of acetaminophen with hydrocodone was found to be more effective than as needed dosing. ${ }^{15}$

Perioperative use of dexamethasone has been shown to improve postoperative vomiting, improve pain control, and decreases airway swelling. ${ }^{16}$ Dexamethasone decreases throat pain and improves time to consumption of oral intake. ${ }^{11}$ Previous studies have raised concerns that steroids may interfere with postoperative hemostasis at concentrations of both 0.05 and $0.5 \mathrm{mg} / \mathrm{kg} .{ }^{17}$ However, more recent meta-analyses have found no overall objective increase in bleeding following tonsillectomy. ${ }^{5}$ Dexamethasone remains a useful adjunct for both pain control and antiemetic effects.

Randomized clinical trials have shown no evidence that intraoperative injection of local anesthetics are effective in improving pain control. ${ }^{11}$ A Cochrane review of perioperative local anesthetic injection showed no improvement in postoperative pain, however, the authors noted the effects may be masked by coadministration of intravenous opioids. ${ }^{18}$ More recent studies have found that peritonsillar dexamethasone infiltration was more effective than local anesthetic or saline in reducing postoperative pain. ${ }^{19}$

Ketamine is a noncompetitive antagonist of N-methyl-Daspartate (NMDA) receptors and can exert analgesic effects. Ketamine has less effect on respiratory drive and airway patency than opioid analgesics. The limitations of ketamine include its psychomimetic effects such as hallucinations and depersonalization, although these effects are not typically seen at low doses. ${ }^{20} \mathrm{~A}$ recent meta-analysis revealed decreased analgesic requirement in subjects who received ketamine preoperatively compared to control without adverse effects such as hallucinations. ${ }^{20}$ The $\mathrm{S}(+)$ ketamine stereoisomer has been shown to have fewer side effects in patients, however, it has not been shown to improve pain control in children. This, however, has not yet been studied in tonsillectomies. Magnesium infusions have been shown to improve pain control following multiple adult surgeries, likely via an NMDA antagonist effect. However, a previous study on pediatric patients showed that an intraoperative magnesium infusion did not attenuate discomfort or emergence delirium following tonsillectomy. ${ }^{21} \mathrm{~A}$ more recent study found that a magnesium bolus followed by infusion was effective in improving emergence delirium, potentially due to an analgesic effect. ${ }^{22}$

\section{Conclusion}

Optimal pain control regimens following pediatric tonsillectomy continue to be a challenge for both anesthesiologists and otorhinolaryngologists. Opioids are associated with significant side effects, and this patient population remains at high risk for opioid-related postoperative respiratory depression. Despite this concern, complete opioid sparing techniques are not likely to provide adequate analgesia. Adjuncts such as IV ibuprofen, IV acetaminophen, dexamethasone, and NMDA antagonists may be safely used in the perioperative period and may have narcotic sparing effects as well as costeffectiveness. Further research into additional analgesics such as IV lidocaine and peritonsillar infiltration adjuncts may provide additional insight.

\section{Disclosure}

The authors report no conflicts of interest in this work.

\section{References}

1. Cullen KA, Hall MJ, Golosinskiy A. Ambulatory surgery in the United States. Natl Health Stat Report. 2009;28(11):1-25.

2. Mattos JL, Robison JG, Greenberg J, Yellon RF. Acetaminophen plus ibuprofen versus opioids for treatment of post-tonsillectomy pain in children. Int J Pediatr Otorhinolaryngol. 2014;78(10):1671-1676.

3. Finley GA, Chorney J, Campbell L. Not small adults: the emerging role of pediatric pain services. Can J Anesth. 2014;61(2):180-187.

4. Subramanyam R, Varughese A, Kurth CD, Eckman MH. Costeffectiveness of intravenous acetaminophen for pediatric tonsillectomy. Pediatr Anaesth. 2014;24(5):467-475.

5. Constant I, Ayari Khalfallah S, Brunaud A, et al. How to replace codeine after tonsillectomy in children under 12 years of age? Guidelines of the French Oto-Rhino-Laryngology - Head and Neck Surgery Society (SFORL). Eur Ann Otorhinolaryngol Head Neck Dis. 2014;131(4):233-238.

6. Lauder G, Emmott A. Confronting the challenges of effective pain management in children following tonsillectomy. Int $J$ Pediatr Otorhinolaryngol. 2014;78(11):1813-1827.

7. Lam J, Woodall KL, Solbeck P, et al. Codeine-related deaths: The role of pharmacogenetics and drug interactions. Forensic Sci Int. 2014;239:50-56. 
8. Racoosin, JA, et al. New Evidence about an Old Drug - Risk with Codeine after Adenotonsillectomy. New England Journal of Medicine. 2013;368(23):2155-2157.

9. Kelly LE, Sommer DD, Ramakrishna J, et al. Morphine or ibuprofen for post-tonsillectomy analgesia: a randomized trial. Pediatrics. 2015; 135(2):307-313.

10. Felden L, Walter C, Harder S, et al. Comparative clinical effects of hydromorphone and morphine: a meta-analysis. Br J Anaesth. 2011; 107(3):319-328.

11. Baugh RF, Archer SM, Mitchell RB, et al. Clinical practice guideline: tonsillectomy in children. Otolaryngol Head Neck Surg. 2011; 144(1 Suppl):S1-30.

12. D’Souza JN, Schmidt RJ, Xie L, Adelman JP, Nardone HC. Postoperative nonsteroidal anti-inflammatory drugs and risk of bleeding in pediatric intracapsular tonsillectomy. Int $J$ Pediatr Otorhinolaryngol. 2015;79(9):1472-1476.

13. Moss JR, Watcha MF, Bendel LP, McCarthy DL, Witham SL, Glover CD. A multicenter, randomized, double-blind placebo-controlled, single dose trial of the safety and efficacy of intravenous ibuprofen for treatment of pain in pediatric patients undergoing tonsillectomy. Pediatric Anesth. 2014;24(5):483-489.

14. Alhashemi JA, Daghistani MF. Effects of intraoperative i.v. acetaminophen vs i.m. meperidine on post-tonsillectomy pain in children. $\mathrm{Br} J$ Anaesth. 2006;96(6):790-795.

15. Gurnaney H, Ganesh A. A randomized clinical trial of the efficacy of scheduled dosing of acetaminophen and hydrocodone for the management of postoperative pain in children after tonsillectomy. Clin J Pain. 2011;27(1):89.
16. Gallagher LQ, Hill C, Ojha S, et al. Perioperative dexamethasone administration and risk of bleeding following tonsillectomy in children: a randomized controlled trial. JAMA. 2012;308(12):1221-1226.

17. Czarnetzki C, Elia N, Lysakowski C, et al. Dexamethasone and risk of nausea and vomiting and postoperative bleeding after tonsillectomy in children: a randomized trial. JAMA. 2008;300(22):2621-2630.

18. Hollis LJ, Burton MJ, Millar JM. Perioperative local anaesthesia for reducing pain following tonsillectomy. Cochrane Database Syst Rev. 2000(2):1-14.

19. Aysenur D, Mine C, Ozgur Y, et al., Pre-emptive peritonsillar dexamethasone vs. levobupivacaine infiltration for relief of postadenotonsillectomy pain in children: a controlled clinical study. Int J Pediatr Otorhinolaryngol. 2014;78(9):1467-1471.

20. Dahmani S, Michelet D, Abback PS, et al. Ketamine for perioperative pain management in children: a meta-analysis of published studies. Pediatric Anaesth. 2011;21(6):636-652.

21. Apan A, Aykaç E, Kazkayasi M, Doganci N, Tahran FD. Magnesium sulphate infusion is not effective on discomfort or emergence phenomenon in paediatric adenoidectomy/tonsillectomy. Int J Pediatr Otorhinolaryngol. 2010;74(12):1367-1371.

22. Abdulatif M, Ahmed A, Mukhtar A, Badawy S. The effect of magnesium sulphate infusion on the incidence and severity of emergence agitation in children undergoing adenotonsillectomy using sevoflurane anaesthesia. Anaesthesia. 2013;68(10):1045-1052.
Ambulatory Anesthesia

\section{Publish your work in this journal}

Ambulatory Anesthesia is an international, peer reviewed, open access journal publishing articles that address all aspects of ambulatory anesthesia practice, in particular: anesthetic techniques, sedation and safety practices, pharmacokinetics, preoperative evaluation, analgesia interventions, regulatory and compliance issues, postoperative recovery,

\section{Dovepress}

patient satisfaction, administrative topics, and cost analysis themes. The manuscript management system is completely online and includes a very quick and fair peer review system, which is all easy to use. Visit http://www.dovepress.com/testimonials.php to read real quotes from published authors. 